\title{
A NEW CLASS OF MODULAR EQUATION FOR WEBER FUNCTIONS
}

\author{
WILLIAM B. HART
}

\begin{abstract}
We describe the construction of a new type of modular equation for Weber functions. These bear some relationship to Weber's modular equations of irrational kind. Numerous examples of these equations are explicitly computed. We also obtain some modular equations of irrational kind which Weber was not able to compute.
\end{abstract}

\section{INTRODUCTION}

A modular equation, in its simplest form, is a polynomial identity relating a modular function $f(\tau)$ of some level $n$ with the function $f(m \tau)$ for some $m \in \mathbb{N}$ (most commonly with $(m, n)=1)$. This $m$ is called the degree of the modular equation.

The basic example of such an identity comes from the Klein $j$-function, where for each $m \in \mathbb{N}$ one has a symmetric polynomial $D_{m}(X, Y) \in \mathbb{Z}[X, Y]$, such that $D_{m}(j(\tau), j(m \tau))=0$ is an identity for all $\tau$ in the complex upper half plane (see [6] for details).

A second example is given by Schläfli modular equations. These provide identities for the Weber functions

$$
\mathfrak{f}(\tau)=\frac{e^{-\frac{\pi i}{24}} \eta\left(\frac{\tau+1}{2}\right)}{\eta(\tau)}, \quad \mathfrak{f}_{1}(\tau)=\frac{\eta\left(\frac{\tau}{2}\right)}{\eta(\tau)}, \quad \mathfrak{f}_{2}(\tau)=\sqrt{2} \frac{\eta(2 \tau)}{\eta(\tau)},
$$

where $\eta(\tau)$ is the well known Dedekind eta function.

We let $u(\tau)$ be any one of these functions and define $v(\tau)=u(m \tau)$ for any $m$ with $(m, 2)=1$. Then if we let $P(\tau)=u(\tau) v(\tau)$ and $Q(\tau)=u(\tau) / v(\tau)$ be the product and quotient of these functions, a Schläfli modular equation is a polynomial identity relating

$$
A=P^{k}+\left(\frac{2}{P}\right)^{k} \text { and } B=Q^{l} \pm\left(\frac{1}{Q}\right)^{l},
$$

where the sign in $B$ and the powers $k, l \in \mathbb{N}$ depend on the degree $m$. After clearing denominators, Schläfli modular equations yield polynomial identities of the kind that we defined above, where $f(\tau)$ is taken to be $u(\tau)$ (see [10] $\S 73$ ).

Generalizations of Schläfli modular equations exist for the higher "level" functions $u_{n}(\tau)=\eta(\tau / n) / \eta(\tau)$ (see [9]).

Other kinds of modular equations exist which are not so easily expressed in terms of the Dedekind eta function. These are expressed in terms of relationships between complete elliptic integrals

$$
K=\int_{0}^{1} \frac{1}{\sqrt{\left(1-y^{2}\right)\left(1-k^{2} y^{2}\right)}} d y .
$$

We let $K, K^{\prime}, L, L^{\prime}$ denote complete elliptic integrals of the first kind with respective moduli $k, k^{\prime}=\sqrt{1-k^{2}}, l$ and $l^{\prime}=\sqrt{1-l^{2}}$ (with $k, l \in(0,1)$ ). Supposing we 
demand

$$
n \frac{K^{\prime}}{K}=\frac{L^{\prime}}{L},
$$

for some $n \in \mathbb{N}$, then some kind of relation between $k$ and $l$ must be induced. By the theory of elliptic integrals, it is possible to show that this relationship is algebraic.

It is common to denote $\alpha=k^{2}$ and $\beta=l^{2}$. A modular equation in this context is then defined to be the algebraic relationship induced between $\alpha$ and $\beta$. One says that $\beta$ has degree $n$ over $\alpha$ (see [2] or Berndt's book [1] for details on this notation and the link to class invariants and the work of Ramanujan).

Any of the above modular equations involving the Weber functions $\mathfrak{f}, \mathfrak{f}_{1}, \mathfrak{f}_{2}$ can be transformed into this alternative form by making use of

$$
\begin{gathered}
\mathfrak{f}(\tau)=2^{1 / 6}\{\alpha(1-\alpha)\}^{-1 / 24}, \quad \mathfrak{f}(m \tau)=2^{1 / 6}\{\beta(1-\beta)\}^{-1 / 24}, \\
\mathfrak{f}_{1}(\tau)=2^{1 / 6}\left\{\frac{(1-\alpha)^{2}}{\alpha}\right\}^{1 / 24}, \quad \mathfrak{f}_{1}(m \tau)=2^{1 / 6}\left\{\frac{(1-\beta)^{2}}{\beta}\right\}^{1 / 24}, \\
\mathfrak{f}_{2}(\tau)=2^{1 / 6}\left\{\frac{\alpha^{2}}{(1-\alpha)}\right\}^{1 / 24}, \quad \mathfrak{f}_{2}(m \tau)=2^{1 / 6}\left\{\frac{\beta^{2}}{(1-\beta)}\right\}^{1 / 24},
\end{gathered}
$$

where $\tau=\frac{i K^{\prime}}{K}$ and $\beta$ is of degree $m$ over $\alpha$. These relations can be derived from (10) of $\S 34$ and (10) and (12) of $\S 42$ in Weber's Lehrbuch [10].

In particular, Schläfli modular equations for $\mathfrak{f}$ become relations between the functions

$$
P=2^{1 / 3}\{\alpha(1-\alpha) \beta(1-\beta)\}^{-1 / 24} \text { and } Q=\left\{\frac{\alpha(1-\alpha)}{\beta(1-\beta)}\right\}^{-1 / 24} .
$$

A Russel type modular equation (see [3] and [5]) can be defined as a polynomial relation between functions

$$
x=(\alpha \beta)^{s / 8} \text { and } y=\{(1-\alpha)(1-\beta)\}^{s / 8},
$$

for some $s \in \mathbb{N}$ depending on the degree of $\beta$ over $\alpha$. Modular equations of this kind are also called irrational modular equations after the fact that, in terms of the function $k^{2}$, the functions $x$ and $y$ can be expressed by taking successive square roots. Russel called these equations $k \lambda-k^{\prime} \lambda^{\prime}$ modular equations.

Equations relating $\alpha^{1 / 8}$ and $\beta^{1 / 8}$ are called Jacobi-Sohnke modular equations.

Ramanujan stated numerous modular equations in terms of three functions defined by Russel (see [5])

$$
\begin{gathered}
P=1+(-1)^{(n+1) / 8}\left((\alpha \beta)^{1 / 8}+\{(1-\alpha)(1-\beta)\}^{1 / 8}\right), \\
Q=(\alpha \beta)^{1 / 8}+\{(1-\alpha)(1-\beta)\}^{1 / 8}+(-1)^{(n+1) / 8}\{\alpha \beta(1-\alpha)(1-\beta)\}^{1 / 8}, \\
R=\{\alpha \beta(1-\alpha)(1-\beta)\}^{1 / 8} .
\end{gathered}
$$

Weber also investigated modular equations of this type (see [11]), so that they have been called modular equations of Weber type.

The kind of modular equation we are most interested in, was defined by Weber in his Lehrbuch [10]. He set

$$
\begin{gathered}
2^{k} A=\{\mathfrak{f}(\tau) \mathfrak{f}(m \tau)\}^{k} \pm\left(\left\{\mathfrak{f}_{1}(\tau) \mathfrak{f}_{1}(m \tau)\right\}^{k}+\left\{\mathfrak{f}_{2}(\tau) \mathfrak{f}_{2}(m \tau)\right\}^{k}\right) \\
B= \pm \frac{2^{k}}{\{\mathfrak{f}(\tau) \mathfrak{f}(m \tau)\}^{k}}+\frac{2^{k}}{\left\{\mathfrak{f}_{1}(\tau) \mathfrak{f}_{1}(m \tau)\right\}^{k}}+\frac{2^{k}}{\left\{\mathfrak{f}_{2}(\tau) \mathfrak{f}_{2}(m \tau)\right\}^{k}},
\end{gathered}
$$


where the signs and the value of $k$ depend on the degree $m$. A polynomial relation between functions $A$ and $B$ of this form, he called a modular equation of irrational kind. This is again because a square root is taken when expressing the equation in terms of $k^{2}$ (see $\S 75$ of [10] for details on both the naming and description of these modular equations).

For modular equations involving $\alpha$ and $\beta$ there are variants depending on four moduli $\alpha, \beta, \gamma$ and $\delta$ with $\beta, \gamma$ and $\delta$ having degree $m, m^{\prime}$ and $m m^{\prime}$ over $\alpha$ respectively. Such modular equations are said to be of mixed kind (see [11] and $[5])$.

The modular equation we will introduce in this paper is a variation on the Weber modular equation of irrational kind. It was discovered whilst attempting to make use of Weber's modular equations to provide relations between, and to evaluate, class invariants associated with the Weber functions.

Specifically, we define

$$
\begin{gathered}
C=\{\mathfrak{f}(\tau) \mathfrak{f}(m \tau)\}^{k}, \\
D=\left(\left\{\mathfrak{f}_{1}(\tau) \mathfrak{f}_{2}(m \tau)\right\}^{l}+\left\{\mathfrak{f}_{2}(\tau) \mathfrak{f}_{1}(m \tau)\right\}^{l}\right) / \sqrt{2}^{l},
\end{gathered}
$$

where the values of $k, l \in \mathbb{N}$ depend on the degree $m$.

Notice that the function $C$ is similar to the first term of Weber's function $A$ above, whilst the function $D$ looks similar to the remaining two terms, with the most important difference being that $\mathfrak{f}_{1}(m \tau)$ and $\mathfrak{f}_{2}(m \tau)$ have been interchanged. This particular feature turns out to be critical to the utility of these modular equations in the computation of class invariants. In the case of Weber's own modular equations computation of class invariants turns out to be very difficult except in the simplest of cases. The algorithm for computing class invariants using the new modular equations described here will be given in a forthcoming paper, [7].

\section{Constructing the Modular Equations}

The general principle that we use to construct our modular equations is that if one has a modular function $f(\tau)$ for some subgroup $\Gamma^{\prime}$ of the modular group (or even a discrete subgroup of $\mathrm{SL}_{2}(\mathbb{R})$ ) and the function has a zero at each cusp (i.e. its $q$-expansion at each cusp vanishes up to and including the constant term) and it has no poles on the complex upper half plane, then the function is identically zero.

This is a simple consequence of the fact that the function $f(\tau)$, being invariant under the action of $\Gamma^{\prime}$, can be thought of as a function on the Riemann surface obtained by compactifying the upper half plane modulo the action of $\Gamma^{\prime}$. Since it has no poles anywhere on this compact Riemann surface, the function must be a constant. Since the function is zero at the cusps, that constant must be zero.

Thus, our first instinct is to find some polynomial combination $F(C, D)$ of the functions $C$ and $D$ defined in the introduction, whose $q$-expansion vanishes, hoping that $F(C, D)=0$ is then an identity. Indeed, our functions are constructed from the Dedekind eta function which has no zeroes or poles in the complex upper half plane, and so both $C$ and $D$ have no poles in the upper half plane. However, the problem with this naive approach is that $C$ and $D$ are not functions for a group with a small number of cusps. The level of these functions may in fact be quite high, and the number of cusps also large. Thus it becomes computationally prohibitive to check the $q$-expansion at each of these cusps to be sure any purported polynomial identity $F(C, D)=0$ holds.

The way around this problem is to construct $G=\prod_{i} F_{i}$ where the $F_{i}$ form a set of transforms of the function $F(C, D)$ under the action of a group $\Gamma^{\prime}$ which has far 
fewer cusps. Then the function $G$ will also satisfy the conditions given above, so that $G=0$ is an identity.

We will construct the transforms $F_{i}$ in such a way that $G=0$ will imply the vanishing of all the factors $F_{i}$, including $F(C, D)$. The identity $F(C, D)=0$ is, of course, our modular equation.

The particular group that we will end up using will only have two inequivalent cusps (at $i \infty$ and 1). Because the $q$-series at both of these cusps will be related, our computational efforts will be limited to checking that the $q$-series of $G$ vanishes at $i \infty$.

In order to do this we will find a polynomial $F$, using a computer algebra system, such that the $q$-series at $i \infty$ of $F(C, D)$ vanishes at $i \infty$. It will turn out that the $q$-series of all the transforms $F_{i}$ of $F$ will be related in such a way that if the $q$-series of one vanishes at $i \infty$ then all of them do.

Because $G$ will be invariant under the transformation $\tau \rightarrow-1 / \tau$, the $q$-series at 0 of $G$ vanishes if and only if it does at $i \infty$. But the $q$-series at the cusp 1 is related to that at 0 since one can be obtained from the other via the transformation $\tau \rightarrow \tau-1$. However, this only has the effect of mutiplying the coefficients of the $q$-series by roots of unity. Thus one vanishes if and only if the other does.

In summary, the above argument shows that if we can define our functions $C$ and $D$ in a way that satisfies all the different criteria set out in the foregoing, all that will be required in any particular case to find a modular equation will be to find a polynomial $F(X, Y)$ such that the $q$-series of $F(C, D)$ vanishes at $i \infty$. Then the above argument shows that $G$ vanishes at all cusps and thus $G=0$ is an identity. This in turn implies one of the $F_{i}$ is identically zero. In Section 1.2.4 we then argue that all the related $F_{i}$ are identically zero, including $F(C, D)$. The identity $F(C, D)=0$ is then our modular equation.

The remainder of Section 1 is dedicated to describing the congruence group which we will make use of throughout the paper, to defining functions $C$ and $D$, for each degree, that satisfy all the requirements above, and to filling in the above-mentioned technical part of our argument that we have deferred until Section 1.2.4.

Finally in Section 2, we actually compute some modular equations by finding the explicit polynomials $F(X, Y)$ that make the $q$-series of $F(C, D)$ vanish at $i \infty$.

1.1. The Congruence Subgroup $\Gamma^{(4)}$. It is sometimes possible to obtain a simpler theory of modular equations by working with a congruence subgroup of a lower level, but at the expense of not obtaining the tightest possible modular equations. Although this makes computations quite simple, there is a tradeoff between theoretical complexity, and the simplicity of the modular equations obtained. In our case, to obtain the best possible modular equations, it turns out to be prudent to work with a congruence subgroup of level 4 .

This group, which we use throughout our computations, will be denoted $\Gamma^{(4)}$. It has as generators $T^{4}, S, T^{2} S T^{-2}$, where, as usual, by $T$ and $S$ we mean the elements of $\mathrm{PSL}_{2}(\mathbb{Z})$ corresponding to transformations $\tau \rightarrow \tau+1$ and $\tau \rightarrow-1 / \tau$ of the complex upper half plane, and associated to images of the matrices $\left(\begin{array}{ll}1 & 1 \\ 0 & 1\end{array}\right)$ and $\left(\begin{array}{cc}0 & -1 \\ 1 & 0\end{array}\right)$ in $\operatorname{PSL}_{2}(\mathbb{Z})$

The following diagram shows a fundamental domain for $\Gamma^{(4)}$ along with its boundary transformations, showing that the group has index 6 in the full modular group, and 
that it has two inequivalent cusps $\{1, i \infty\}$ (note the cusps at 1 and 3 are equivalent under the transformation $T^{2} S T^{-2}$ ).

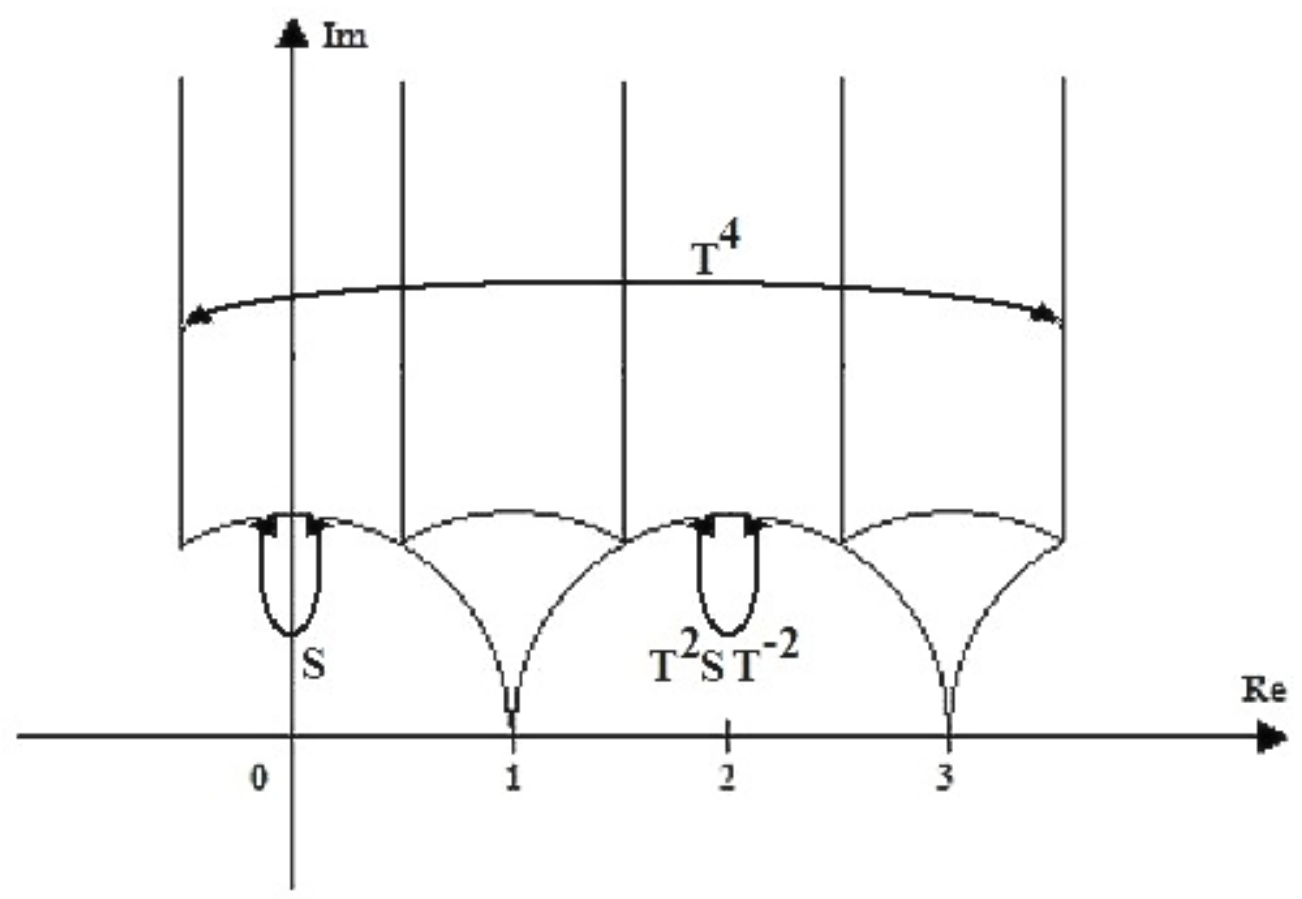

The group $\Gamma^{(4)}$ is a conjugate of $\Gamma^{0}(4)$ and therefore is of level 4 .

1.2. The Function Theory. We have defined the functions $C$ and $D$, that we will use, in the introduction (up to specifying $k$ and $l$ ). Now we need to construct transforms of these under the action of $\Gamma^{(4)}$.

In particular, we define, for any fixed degree $m \in \mathbb{N}$, with $m$ odd, the set of functions

$$
\begin{gathered}
C_{a, b, d}=\left\{\mathfrak{f}(\tau) \mathfrak{f}\left(\frac{a \tau+b}{d}\right)\right\}^{k} \\
D_{a, b, d}=\left(\left\{\mathfrak{f}_{1}(\tau) \mathfrak{f}_{2}\left(\frac{a \tau+b}{d}\right)\right\}^{l}+\left\{\mathfrak{f}_{2}(\tau) \mathfrak{f}_{1}\left(\frac{a \tau+b}{d}\right)\right\}^{l}\right) / \sqrt{2}^{l},
\end{gathered}
$$

for each triple $a, b, d$ such that $a d=m$, and $b$ traversing a complete set of equivalence classes modulo $d$. We will also require that $0 \leq b<16 d$ and that $b$ is divisible by 16. The values $k, l \in \mathbb{N}$ will be fixed in the sequel.

Note that with this notation, our original functions $C$ and $D$ have become $C_{m, 0,1}$ and $D_{m, 0,1}$.

1.2.1. Linear Transformations of $C_{a, b, d}$ and $D_{a, b, d}$. In order to show that the functions we have defined are permuted by $\Gamma^{(4)}$, we must first determine what happens to $\frac{a \tau+b}{d}$ when we apply each of the generators, $T^{4}, S, T^{2} S T^{-2}$, of $\Gamma^{(4)}$. Writing such a generator as $A=\left(\begin{array}{ll}\alpha & \beta \\ \gamma & \delta\end{array}\right)$ we will solve the matrix equation

$$
\left(\begin{array}{ll}
a & b \\
0 & d
\end{array}\right)\left(\begin{array}{ll}
\alpha & \beta \\
\gamma & \delta
\end{array}\right)=\left(\begin{array}{ll}
\alpha^{\prime} & \beta^{\prime} \\
\gamma^{\prime} & \delta^{\prime}
\end{array}\right)\left(\begin{array}{ll}
a^{\prime} & b^{\prime} \\
0 & d^{\prime}
\end{array}\right) \text {, }
$$


where $\left(\begin{array}{ll}\alpha^{\prime} & \beta^{\prime} \\ \gamma^{\prime} & \delta^{\prime}\end{array}\right) \in \Gamma=\mathrm{SL}_{2}(\mathbb{Z})$. For if such an equation holds, then $\tau \rightarrow A \tau$ takes $\frac{a \tau+b}{d}$ to $\frac{a^{\prime} \tau+b^{\prime}}{d^{\prime}}$ up to some transformation in $\Gamma$.

The matrix equation leads to the following five equations, which must be solved simultaneously:

$$
\begin{gathered}
\alpha a+\gamma b=\alpha^{\prime} a^{\prime}, \\
\beta a+\delta b=\alpha^{\prime} b^{\prime}+\beta^{\prime} d^{\prime}, \\
\gamma d=\gamma^{\prime} a^{\prime}, \\
\delta d=\gamma^{\prime} b^{\prime}+\delta^{\prime} d^{\prime}, \\
\alpha^{\prime} \delta^{\prime}-\beta^{\prime} \gamma^{\prime}=1 .
\end{gathered}
$$

The generators $S$ and $T^{2} S T^{-2}$ of $\Gamma^{(4)}$ have $\alpha$ and $\delta$ even, but $T^{4}$ does not. However, in $\mathrm{PSL}_{2}(\mathbb{Z}), T^{4}$ and $S\left(S T^{4}\right)$ are the same transformation, and both $S$ and $S T^{4}$ have $\alpha$ and $\delta$ even. Thus $\Gamma^{(4)}$ has generators which all have $\alpha$ and $\delta$ even (namely $S$, $S T^{4}$ and $\left.T^{2} S T^{-2}\right)$.

The matrices corresponding to these generators are

$$
S:\left(\begin{array}{cc}
0 & -1 \\
1 & 0
\end{array}\right), \quad S T^{4}:\left(\begin{array}{cc}
0 & -1 \\
1 & 4
\end{array}\right), \quad T^{2} S T^{-2}:\left(\begin{array}{cc}
2 & -5 \\
1 & -2
\end{array}\right) .
$$

Since we only define functions for triples $a, b, d$ with $16 \mid b$, then in the five equations above, we have that $16 \mid b$ and $16 \mid b^{\prime}$. Furthermore in our definition of $C$ and $D$ we specified that $m$ is odd, so $a, d, a^{\prime}$ and $d^{\prime}$ are all odd. Thus from equation $(7), \delta^{\prime}$ is even, which because of equation (8), implies that $\gamma^{\prime}$ and $\beta^{\prime}$ are odd. Finally, equation (4) implies that $\alpha^{\prime}$ is even.

Since we will be interested in transformations of the functions $\mathfrak{f}\left(\frac{a \tau+b}{d}\right), \mathfrak{f}_{1}\left(\frac{a \tau+b}{d}\right)$ and $\mathfrak{f}_{2}\left(\frac{a \tau+b}{d}\right)$, the above implies that we require transformation formulae for the functions $\mathfrak{f}, \mathfrak{f}_{1}$ and $\mathfrak{f}_{2}$ under the transformation $\tau \rightarrow \frac{\alpha^{\prime} \tau+\beta^{\prime}}{\gamma^{\prime} \tau+\delta^{\prime}}$. Note that given the parities we have just computed, $\delta^{\prime}+\gamma^{\prime}+\beta^{\prime}-\alpha^{\prime}, \alpha^{\prime}$ and $\delta^{\prime}$ are all even. Transformation formulae for the Weber functions under these conditions are given in $\S 40$ of [10]. We repeat them here for the convenience of the reader.

For $\delta^{\prime}+\gamma^{\prime}+\beta^{\prime}-\alpha^{\prime}$ even,

$$
\mathfrak{f}\left(\frac{\alpha^{\prime} \tau+\beta^{\prime}}{\gamma^{\prime} \tau+\delta^{\prime}}\right)=\left(\frac{2}{\delta^{\prime}-\gamma^{\prime}}\right) \sigma^{\prime} \zeta_{16}^{-3\left[\left(\delta^{\prime}-\gamma^{\prime}\right)\left(\delta^{\prime}+\gamma^{\prime}+\beta^{\prime}-\alpha^{\prime}\right)\right]} \mathfrak{f}(\tau),
$$

where $\sigma^{\prime}=\zeta_{3}^{-\left[\delta^{\prime}\left(\beta^{\prime}-\gamma^{\prime}\right)-\left(\delta^{2}-1\right) \alpha^{\prime} \gamma^{\prime}\right]}$.

For $\alpha^{\prime}$ even,

$$
\mathfrak{f}_{1}\left(\frac{\alpha^{\prime} \tau+\beta^{\prime}}{\gamma^{\prime} \tau+\delta^{\prime}}\right)=\left(\frac{2}{\beta^{\prime}}\right) \sigma^{\prime} \zeta_{16}^{-3\left[\beta^{\prime}\left(2 \delta^{\prime}-\alpha^{\prime}\right)\right]} \mathfrak{f}_{2}(\tau),
$$

with the same $\sigma^{\prime}$ as in the previous case.

For $\delta^{\prime}$ even,

$$
\mathfrak{f}_{2}\left(\frac{\alpha^{\prime} \tau+\beta^{\prime}}{\gamma^{\prime} \tau+\delta^{\prime}}\right)=\left(\frac{2}{\gamma^{\prime}}\right) \sigma^{\prime} \zeta_{16}^{3\left[\gamma^{\prime}\left(2 \alpha^{\prime}-\delta^{\prime}\right)\right]} \mathfrak{f}_{1}(\tau),
$$

with the same $\sigma^{\prime}$ again. 
It turns out that, in order to make further progress towards obtaining modular equations for our functions as defined in (3) for $m$ odd, it is necessary to choose $l$ even. This has two immediate consequences. Firstly, the Jacobi symbols in the transformation formulae for $\mathfrak{f}_{1}$ and $\mathfrak{f}_{2}$, that we have just quoted, become irrelevant. Secondly, the 16-th roots of unity appearing in those transformation formulae become 8-th roots of unity, whose values only depend on $\alpha^{\prime}, \beta^{\prime}, \gamma^{\prime}$ and $\delta^{\prime}$ modulo 8 . But these latter values are derived from equations (4) through (7) above, which become (since $16 \mid b$ and $16 \mid b^{\prime}$ )

$$
\begin{aligned}
& \alpha a \equiv \alpha^{\prime} a^{\prime} \quad(\bmod 16), \\
& \beta a \equiv \beta^{\prime} d^{\prime} \quad(\bmod 16), \\
& \gamma d \equiv \gamma^{\prime} a^{\prime} \quad(\bmod 16), \\
& \delta d \equiv \delta^{\prime} d^{\prime} \quad(\bmod 16) .
\end{aligned}
$$

Making use of these equivalences, the eighth roots of unity that we are after can now be seen to depend on the values of the following, modulo 8 .

$$
\begin{aligned}
& X=\frac{\beta a}{d^{\prime}}\left(2 \frac{\delta d}{d^{\prime}}-\frac{\alpha a}{a^{\prime}}\right), \\
& Y=\frac{\gamma d}{a^{\prime}}\left(2 \frac{\alpha a}{a^{\prime}}-\frac{\delta d}{d^{\prime}}\right) .
\end{aligned}
$$

Now we make use of the fact that for any $a$ odd, $a^{2} \equiv 1(\bmod 8)\left(\right.$ and thus $a \equiv \frac{1}{a}$ $(\bmod 8))$, the fact that $a d=m=a^{\prime} d^{\prime}$ and the fact that $a, d, a^{\prime}$ and $d^{\prime}$ are all odd. We then find (after some fiddly but trivial computation) that

$$
\begin{gathered}
X \equiv-2 m \beta \delta+\alpha \beta m \quad(\bmod 8) \\
Y \equiv 2 m \alpha \gamma-\gamma \delta m \quad(\bmod 8)
\end{gathered}
$$

Now since $\alpha$ and $\delta$ are even, and $\beta$ and $\gamma$ are odd, we can use directly the formulae (10) and (11), with $\alpha^{\prime}, \beta^{\prime}, \gamma^{\prime}, \delta^{\prime}$ replaced with $\alpha, \beta, \gamma, \delta$ and the corresponding $\sigma^{\prime}$ written $\sigma$, to determine the effect of the transformation $\tau \rightarrow \frac{\alpha \tau+\beta}{\gamma \tau+\delta}$ on the functions $\mathfrak{f}_{1}^{2}(\tau)$ and $\mathfrak{f}_{2}^{2}(\tau)$. We determine that the 8 -th roots of unity involved depend on the quantities $-2 \beta \delta+\beta \alpha$ and $2 \alpha \gamma-\delta \gamma$ respectively.

Putting all this information together, we find that applying the transformation $\tau \rightarrow \frac{\alpha \tau+\beta}{\gamma \tau+\delta}$ to the functions $\left\{\mathfrak{f}_{2}(\tau) \mathfrak{f}_{1}\left(\frac{a \tau+b}{d}\right)\right\}^{2}$ and $\left\{\mathfrak{f}_{1}(\tau) \mathfrak{f}_{2}\left(\frac{a \tau+b}{d}\right)\right\}^{2}$ yields

$$
\left(\sigma \sigma^{\prime}\right)^{2} \zeta_{8}^{3 X^{\prime}}\left\{\mathfrak{f}_{1}(\tau) \mathfrak{f}_{2}\left(\frac{a^{\prime} \tau+b^{\prime}}{d^{\prime}}\right)\right\}^{2}
$$

and

$$
\left(\sigma \sigma^{\prime}\right)^{2} \zeta_{8}^{3 Y^{\prime}}\left\{\mathfrak{f}_{2}(\tau) \mathfrak{f}_{1}\left(\frac{a^{\prime} \tau+b^{\prime}}{d^{\prime}}\right)\right\}^{2}
$$

respectively, where

$$
X^{\prime}=-2 m \beta \delta+\alpha \beta m+2 \alpha \gamma-\delta \gamma
$$

and

$$
Y^{\prime}=2 m \alpha \gamma-\gamma \delta m-2 \beta \delta+\beta \alpha
$$


Note that $Y^{\prime} \equiv m X^{\prime}(\bmod 8)\left(\right.$ use that $\left.m^{2} \equiv 1(\bmod 8)\right)$, i.e. $Y^{\prime} \equiv X^{\prime}(\bmod 8)$, given that $X^{\prime}$ and $Y^{\prime}$ are divisible by 4 for each of the generators $S, S T^{4}$ and $T^{2} S T^{-2}$ of $\Gamma^{(4)}$ that we have chosen to work with. In fact we calculate that $X^{\prime} \equiv$ $Y^{\prime} \equiv 0,4,2(m+3)(\bmod 8)$ for the generators $S, S T^{4}, T^{2} S T^{-2}$ respectively.

Now we must determine the effect of the transformation $\tau \rightarrow \frac{\alpha \tau+\beta}{\gamma \tau+\delta}$ on $\mathfrak{f}\left(\frac{a \tau+b}{d}\right)$.

Firstly, we evaluate the Jacobi symbol which appears in the transformation formula $(9)$ :

$$
\left(\frac{2}{\delta^{\prime}-\gamma^{\prime}}\right)=(-1)^{\frac{\left(\delta^{\prime}-\gamma^{\prime}\right)^{2}-1}{8}}=\zeta_{16}^{\left(\delta^{\prime}-\gamma^{\prime}\right)^{2}-1}=\zeta_{16}^{-3\left[5\left(\delta^{\prime}-\gamma^{\prime}\right)^{2}-5\right]}
$$

Next we see that the 16 -th root of unity that appears in $(9)$ depends on $Z(\bmod 16)$ where

$$
Z=\left(\gamma^{\prime}-\delta^{\prime}\right)\left(\delta^{\prime}+\gamma^{\prime}+\beta^{\prime}-\alpha^{\prime}\right)=-\delta^{\prime 2}-\delta^{\prime} \beta^{\prime}+\delta^{\prime} \alpha^{\prime}+\gamma^{\prime 2}+\beta^{\prime} \gamma^{\prime}-\alpha^{\prime} \gamma^{\prime}
$$

Now writing $\alpha^{\prime} \delta^{\prime}-1=\beta^{\prime} \gamma^{\prime}$ and making use of (12) through (15) and the fact that $a d=m=a^{\prime} d^{\prime}$, we find

$$
Z \equiv-\left(\frac{\delta d}{d^{\prime}}\right)^{2}-\frac{\delta \beta m}{d^{\prime 2}}+2 \alpha \delta-1+\left(\frac{\gamma d}{a^{\prime}}\right)^{2}-\frac{\alpha \gamma m}{a^{\prime 2}} \quad(\bmod 16)
$$

Now we use that $\alpha$ and $\delta$ are even and so any term involving these only depends on the remaining factors of that term modulo 8. As usual we use the fact that $1 / s \equiv s(\bmod 8)$ for odd $s$. Finally we use that $s^{4} \equiv 1(\bmod 16)\left(\right.$ so that $1 / s^{2} \equiv s^{2}$ $(\bmod 16))$ for odd $s$. Applying these facts, we obtain

$$
Z \equiv-\delta^{2}-m \delta \beta+2 \alpha \delta+\left(a^{\prime} d\right)^{2} \gamma^{2}-m \alpha \gamma-1 \quad(\bmod 16) .
$$

We also determine directly the effect of the transformation $\tau \rightarrow \frac{\alpha \tau+\beta}{\gamma \tau+\delta}$ on the function $\mathfrak{f}(\tau)$. We rewrite (9), replacing $\alpha^{\prime}, \beta^{\prime}, \gamma^{\prime}, \delta^{\prime}$ with $\alpha, \beta, \gamma, \delta$ and $\sigma^{\prime}$ with $\sigma$. Then the 16-th root appearing in the transformation formula is $\zeta_{16}^{3\left[-\delta^{2}-\beta \delta+\alpha \delta+\gamma^{2}+\beta \gamma-\alpha \gamma\right]}$ and the Jacobi symbol $\left(\frac{2}{\delta-\gamma}\right)=\zeta_{16}^{3\left[-5(\delta-\gamma)^{2}+5\right]}$.

Combining all this information and simplifying, we find that applying the transformation $\tau \rightarrow \frac{\alpha \tau+\beta}{\gamma \tau+\delta}$ to $\mathfrak{f}(\tau) \mathfrak{f}\left(\frac{a \tau+b}{d}\right)$ yields

$$
\sigma \sigma^{\prime} \zeta_{16}^{3 Z^{\prime}} \mathfrak{f}(\tau) \mathfrak{f}\left(\frac{a^{\prime} \tau+b^{\prime}}{d^{\prime}}\right)
$$

where $Z^{\prime}=(m+1)(\alpha \gamma+\beta \delta+6 \gamma \delta)$.

Note that for the generators $S$ and $T^{2} S T^{-2}, Z^{\prime} \equiv 0(\bmod 16)$ whereas for the generator $S T^{4}, Z^{\prime} \equiv 4(m+1)(\bmod 16)$.

We now summarise the information we have just derived in a table. There are two distinct cases: $m \equiv 1$ or $3(\bmod 4)$.

\begin{tabular}{|c|c|c|}
\hline$m \equiv ?(\bmod 4)$ & $X^{\prime} \equiv Y^{\prime} \equiv ?(\bmod 8)$ & $Z^{\prime} \equiv ?(\bmod 16)$ \\
\hline 1 & $0,4,0$ & $0,8,0$ \\
\hline 3 & $0,4,4$ & $0,0,0$ \\
\hline
\end{tabular}

where three numbers in a row, in each case, correspond to the values of the given quantity after transformation by $S, S T^{4}$ and $T^{2} S T^{-2}$ respectively. 
1.2.2. Invariant Functions. We now try to choose the constants $k$ and $l$ in the functions $C_{a, b, d}$ and $D_{a, b, d}$, so that symmetric combinations of these are invariant under the action of $\Gamma^{(4)}$. This is equivalent to choosing the constants so that the set of $C_{a, b, d}$ and the set of $D_{a, b, d}$ are both permuted (in an equivariant way) under the action of $\Gamma^{(4)}$. In fact we don't quite achieve this, but instead use various combinations of our functions to do something similar.

From $(25),(18),(19)$ and the table above, we can see how the functions $C_{a, b, d}$ and $D_{a, b, d}$ transform. There are two cases:

Case 1: If $m \equiv 1(\bmod 4)$ we choose $k$ to be any natural number and $l$ to be even, in (3). Then $D_{a, b, d} \rightarrow \pm\left(\sigma \sigma^{\prime}\right)^{l} D_{a^{\prime}, b^{\prime}, d^{\prime}}$ and $C_{a, b, d} \rightarrow \pm\left(\sigma \sigma^{\prime}\right)^{k} C_{a^{\prime}, b^{\prime}, d^{\prime}}$ by a transformation from $\Gamma^{(4)}$.

Case 2: If $m \equiv 3(\bmod 4)$, we choose $k$ to be any natural number and $l$ to be divisible by 4 . Then $D_{a, b, d} \rightarrow\left(\sigma \sigma^{\prime}\right)^{l / 4} D_{a^{\prime}, b^{\prime}, d^{\prime}}$ and $C_{a, b, d} \rightarrow\left(\sigma \sigma^{\prime}\right)^{k} C_{a^{\prime}, b^{\prime}, d^{\prime}}$ by a transformation from $\Gamma^{(4)}$.

In both cases, although the functions aren't quite permuted by our transformations, they nearly are, and this will turn out to be enough.

For convenience, in the sequel, we will settle on fixed values of $k$ and $l$ in our definition of the functions $C_{a, b, d}$ and $D_{a, b, d}$. Although this is not necessary to our theory, it is convenient and will fix the definition of the functions $C_{a, b, d}$ and $D_{a, b, d}$ as per the following table.

\begin{tabular}{|c|c|c|}
\hline$m \equiv ? \quad(\bmod 4)$ & $C_{a, b, d}$ & $D_{a, b, d}$ \\
\hline 1 & $\mathfrak{f}(\tau) \mathfrak{f}\left(\frac{a \tau+b}{d}\right)$ & $\frac{1}{2}\left\{\mathfrak{f}_{1}(\tau) \mathfrak{f}_{2}\left(\frac{a \tau+b}{d}\right)\right\}^{2}+\frac{1}{2}\left\{\mathfrak{f}_{2}(\tau) \mathfrak{f}_{1}\left(\frac{a \tau+b}{d}\right)\right\}^{2}$ \\
\hline 3 & $\mathfrak{f}(\tau) \mathfrak{f}\left(\frac{a \tau+b}{d}\right)$ & $\frac{1}{4}\left\{\mathfrak{f}_{1}(\tau) \mathfrak{f}_{2}\left(\frac{a \tau+b}{d}\right)\right\}^{4}+\frac{1}{4}\left\{\mathfrak{f}_{2}(\tau) \mathfrak{f}_{1}\left(\frac{a \tau+b}{d}\right)\right\}^{4}$ \\
\hline
\end{tabular}

where $a, b, d$ satisfy $a d=m, b$ traverses a complete set of residues modulo $d$, $0 \leq b<16 d$ and $16 \mid b$.

At this point, we have enough information to construct modular equations for these functions. However, with just a little more work, we can obtain much simpler modular equations in certain cases. What is missing is an analysis of the cubed roots of unity, $\sigma$ and $\sigma^{\prime}$.

In the case where $m$ is not divisible by 3 , we can place an additional restriction on our choice of representatives $a, b, d$, namely that $3 \mid b$ (we now allow $0 \leq b<48 d$ ). When this is the case, the equations (4) through (7) become

$$
\begin{gathered}
\alpha a \equiv \alpha^{\prime} a^{\prime} \quad(\bmod 3), \\
\beta a \equiv \beta^{\prime} d^{\prime} \quad(\bmod 3), \\
\gamma d=\gamma^{\prime} a^{\prime}, \\
\delta d \equiv \delta^{\prime} d^{\prime} \quad(\bmod 3) .
\end{gathered}
$$

In particular, from these equations, if $m=a d \equiv 2(\bmod 3)$, so that one of $a$ and $d$ is 1 modulo 3 whilst the other is 2 modulo 3 , we see that one of two possibilities can occur. Either $\alpha \equiv \alpha^{\prime}, \delta \equiv \delta^{\prime}, \beta \equiv-\beta^{\prime}, \gamma \equiv-\gamma^{\prime}(\bmod 3)$ or $\alpha \equiv-\alpha^{\prime}, \delta \equiv-\delta^{\prime}$, $\beta \equiv \beta^{\prime}, \gamma \equiv \gamma^{\prime}(\bmod 3)$.

In either case, by examining the generators $S, S T^{4}$ and $T^{2} S T^{-2}$ (in which either $\alpha \equiv 0$ or $\delta \equiv 1(\bmod 3)$ in each case), we see that $\sigma \sigma^{\prime}=1$ (recall the definition of $\sigma^{\prime}$ after (9), $\sigma$ being defined similarly, but in terms of $\left.\alpha, \beta, \gamma, \delta\right)$. 
When $m \equiv 1(\bmod 3)$ we do not have $\sigma \sigma^{\prime}=1$ in general so $m \equiv 2(\bmod 3)$ remains the only case where we are able to achieve a simplification. Indeed, in this case the modular equations we obtain are significantly less cumbersome because of this simplification.

1.2.3. Modular polynomials. The next step in constructing modular equations is to consider polynomial expressions $F_{a, b, d}=F\left(D_{a, b, d}, C_{a, b, d}\right)$ of the two functions $D_{a, b, d}, C_{a, b, d}$ for a fixed polynomial $F(X, Y) \in \mathbb{Z}[X, Y]$.

In the case that $m \equiv 1(\bmod 4)$, the $C_{a, b, d}$ and $D_{a, b, d}$ are permuted up to the same sixth roots of unity, unless $m \equiv 2(\bmod 3)$, in which case they are permuted up to a factor of \pm 1 . Thus, we should choose our polynomial $F$ such that every monomial $X^{a} Y^{b}$ has the same value for $a-b(\bmod 6)$, unless we have $m \equiv 2(\bmod 3)$, in which case it suffices to have $a-b(\bmod 2)$ the same for each monomial. If these choices are made, then the functions $F_{a, b, d}$ will be permuted up to roots of unity by transformations in $\Gamma^{(4)}$. Thus some power of the product $G=\prod_{a, b, d} F_{a, b, d}$ is invariant under the action of $\Gamma^{(4)}$.

Similarly in the case that $m \equiv 3(\bmod 4)$ we choose the polynomial $F$ such that every monomial has the same value for $a+b(\bmod 3)$, except when $m \equiv 2(\bmod 3)$, in which case we can let $a$ and $b$ be arbitrary natural numbers. In these cases, the functions $F_{a, b, d}$ will be permuted by $\Gamma^{(4)}$ upto roots of unity, as for the other case.

We summarise the conditions on the monomials of the polynomial $F(X, Y)$ in the following table.

\begin{tabular}{|c|cc|}
\hline$m \equiv \quad(\bmod 12)$ & \multicolumn{2}{|c|}{$X^{a} Y^{b}$} \\
\hline 1 & same $a-b$ & $(\bmod 6)$ \\
\hline 3 & same $a+b$ & $(\bmod 3)$ \\
\hline 5 & same $a-b$ & $(\bmod 2)$ \\
\hline 7 & same $a+b$ & $(\bmod 3)$ \\
\hline 9 & same $a-b$ & $(\bmod 6)$ \\
\hline 11 & \multicolumn{2}{|c|}{ arbitrary } \\
\hline
\end{tabular}

1.2.4. q-series. In all cases just given, some power of the function $G=\prod_{a, b, d} F_{a, b, d}$ is invariant under the action of the group $\Gamma^{(4)}$. We now wish to construct $G$, by choosing a suitable polynomial $F \in \mathbb{Z}[X, Y]$, such that the $q$-series of $G$ at $i \infty$ vanishes up to and including the constant term.

As mentioned in the introduction, however, in doing all this, we hope to avoid examining the $q$-series of each of the functions $F_{a, b, d}$ individually.

Firstly we consider the case where $m$ is an odd prime. Suppose that we can choose $F$ in such a way that the $q$-series of $F_{m, 0,1}$ vanishes up to an including the constant term. Suppose that we can also show that the $q$-series of the other $F_{a, b, d}$ for this $m$ are related to that of $F_{m, 0,1}$ in such a way that it is implied that their $q$-series vanish in a like manner. Then the $q$-series of $G$ will vanish up to and including the constant term.

So we look for relationships between the $q$-series of the functions $F_{a, b, d}$ for $m$ an odd prime. But since $a d=m$, a complete set of such functions is given by $(a, b, d)$ traversing $\{(m, 0,1),(1,0, m),(1,16, m),(1,32, m), \ldots,(1,16(m-1), m)\}$ (or a simple variation of this if $b$ is to be divisible by 3 ). But it is easy to check from the definition of the functions $D_{m, 0,1}, C_{m, 0,1}$ and $D_{1,0, m}, C_{1,0, m}$ that the $q$-series of $F_{m, 0,1}$ is related to that of $F_{1,0, m}$ by the transformation $\tau \rightarrow \tau / m$, i.e. $q \rightarrow q^{\frac{1}{m}}$. Thus if the $q$-series of $F_{m, 0,1}$ vanishes up to and including the constant term, then so does that of $F_{1,0, m}$. 
Similarly, the $q$-series of $F_{1,0, m}$ is related to that of $F_{1, b, m}$ by the transformation $\tau \rightarrow \tau+b$. However, this transformation only changes the coefficients of the $q$-series by roots of unity. Thus, such a transformation preserves the vanishing property that we are after.

In the case where $m$ is not prime, we are not so lucky in finding transformations which preserve the vanishing property we are after, and which relate all the functions $F_{a, b, d}$. However, all is not lost. In fact, the functions $F_{a, b, d}$ with $(a, b, d) \in\{(m, 0,1),(1,0, m),(1,16, m),(1,32, m), \ldots,(1,16(m-1), m)\}$ (or the relevant variation for $3 \mid b$ ), are still related as before. It is just that we now have additional functions $F_{a, b, d}$ which are not related to any of these by such simple transformations, (in particular for the cases where $a$ and $d$ are non-trivial factors of $m)$.

The trick here is simply to demand that the $q$-series of $F_{m, 0,1}$ vanishes so far past the constant term that the contribution of all the wayward functions $F_{a, b, d}$ to the $q$-series of $G$ is outweighed by the contribution from $F_{m, 0,1}$ and the functions it is related to. In performing computations of modular equations for this paper, we always worked to a $q$-series precision far beyond that required to ensure that the contribution of $F_{m, 0,1}$ and its relatives outweighs the contribution of the other functions $F_{a, b, d}$ to the $q$-series of $G$.

Of course, this requires us to compute the leading power of the $q$-series of each of the wayward $F_{a, b, d}$. However, this is easy enough in practice.

Having verified the $q$-series condition at $i \infty$ the argument given in the introduction also ensures that the $q$-series of $G$ vanishes at the other cusp 1 , on account of the transformation $\tau \rightarrow-1 / \tau$ being in our group $\Gamma^{(4)}$. Thus $G=0$ identically, and this in turn implies that one of the factors $F_{a, b, d}$ of $G$ must be identically zero.

In the case where $m$ is prime, all of the functions $F_{a, b, d}$ are transforms of one another, and so if one vanishes identically, they all must.

In the case where $m$ is not prime, the functions $F_{m, 0,1}$ and $F_{1, b, m}$ are all still transforms of one another and it is easy to test numerically (or by examining the leading terms of the $q$-series of each) that none of the other $F_{a, b, d}$ vanish identically. In either case, $F_{m, 0,1}=F(C, D)=0$ must itself vanish identically, and it is then the modular equation we are looking for.

\section{Computing the Modular Equations}

All that remains is for us to compute actual modular polynomials $F \in \mathbb{Z}[X, Y]$ such that the $q$-series of $F_{m, 0,1}=F\left(C_{m, 0,1}, D_{m, 0,1}\right)$ vanishes sufficiently far. For this purpose, we used the PARI-GP computer algebra system on a single processor desktop computer running at $2.5 \mathrm{GHz}$. This required a considerable amount of operator time, however each of the computations themselves took only seconds to complete when verifying the vanishing of the relevant $q$-series.

For further details on the intricacies of using a computer algebra system to find modular equations, we refer the interested reader to our other paper [9]. Many of the methods used there were similar to the case at hand.

We list the modular equations computed for various values of $m$. We simply write $C$ and $D$ for $C_{m, 0,1}$ and $D_{1,0, m}$ respectively.

$m=3$ :

$$
D-C=0 \text {. }
$$

$m=5$ :

$$
C-D=0 \text {. }
$$


$m=7:$

$$
C^{6}-5 C^{3}-C D^{2}+8=0 .
$$

$m=9$ :

$$
C^{13} D-C^{6} D^{6}+C^{12}-8 C^{5} D^{5}-20 C^{4} D^{4}+80 C^{2} D^{2}+128 C D+64=0 .
$$

$m=11$ :

$$
C^{4}-3 C^{2}-C D+4=0 .
$$

$m=13$ :

$$
C^{12}-8 C^{7} D-C D^{7}-11 C^{4} D^{4}+128 C^{2} D^{2}-256=0 .
$$

$m=15$ :

$$
\begin{gathered}
C^{18} D^{2}-C^{17}+3 C^{15} D^{2}-5 C^{14}+22 C^{12} D^{2}-29 C^{11}-13 C^{10} D^{4}+5 C^{9} D^{2} \\
-40 C^{8}-95 C^{7} D^{4}+176 C^{6} D^{2}-C^{5} D^{6}-64 C^{5}-104 C^{4} D^{4}+192 C^{3} D^{2}+512 D^{2}=0 .
\end{gathered}
$$

$m=17$ :

$$
C^{8}-5 C^{5} D+6 C^{4}-C^{3} D^{3}+6 C^{2} D^{2}-12 C D+8=0 .
$$

$m=19$ :

$$
\begin{gathered}
C^{24}-33 C^{18}+14 C^{17} D-17 C^{16} D^{2}+569 C^{12}-861 C^{11} D+1090 C^{10} D^{2} \\
-386 C^{9} D^{3}+37 C^{8} D^{4}-C^{7} D^{5}-2112 C^{6}+896 C^{5} D-1088 C^{4} D^{2}+4096=0 .
\end{gathered}
$$

As an illustration of our algorithm, we work through the $m=3$ case explicitly.

We expand the Weber functions themselves in terms of the nome $q=e^{\pi i \tau}$. This ultimately allows us to represent the $q$-series of our functions without fractional powers of the nome $q$.

Firstly, the eta function in terms of this nome is

$$
\eta(q)=q^{1 / 12} \prod_{n=1}^{\infty}\left(1-q^{2 n}\right) .
$$

It is convenient to define the function

$$
e(q)=\prod_{n=1}^{\infty}\left(1-q^{n}\right)
$$

which is $\eta\left(q^{1 / 2}\right)$ with the leading fractional power of $q$ omitted. The function $e(q)$ can easily be represented in Pari as a power series in $q$.

Now the Weber functions can be expressed in terms of the function $e(q)$ as follows

$$
\mathfrak{f}(\tau)=q^{-1 / 24} \frac{e(-q)}{e\left(q^{2}\right)}, \quad \mathfrak{f}_{1}(q)=q^{-1 / 24} \frac{e(q)}{e\left(q^{2}\right)}, \mathfrak{f}_{2}(q) / \sqrt{2}=q^{1 / 12} \frac{e\left(q^{4}\right)}{e\left(q^{2}\right)} .
$$

Again, these functions can be represented easily in Pari if the fractional powers of $q$ are omitted for now. In particular, we can represent the functions $f(q)=q^{1 / 24} \mathfrak{f}(q)$, $f_{1}(q)=q^{1 / 24} \mathfrak{f}_{1}(q)$ and $f_{2}(q)=q^{-1 / 12} \mathfrak{f}_{2}(q) / \sqrt{2}$ with integer coefficients and without fractional powers of $q$.

Of course in practice, we truncate our series for $e(q)$ at $O\left(q^{100}\right)$ say, which is a high enough $q$-precision for our purposes (though here we truncate much earlier, to save space).

Now according to Section 1.2.2 we should take $k=1$ and $l=4$ in our definition of the functions $C$ and $D$. Thus these functions become

$$
C(q)=\mathfrak{f}(q) \mathfrak{f}\left(q^{3}\right), \quad D(q)=\frac{1}{4}\left(\mathfrak{f}_{1}(q) \mathfrak{f}_{2}\left(q^{3}\right)\right)^{4}+\frac{1}{4}\left(\mathfrak{f}_{2}(q) \mathfrak{f}_{1}\left(q^{3}\right)\right)^{4} .
$$


Ignoring the factor $q^{-1 / 6}$ in the case of $C(q)$ we can represent this function in Pari by $C=f(q) f\left(q^{3}\right)$. Similarly ignoring a factor of $q^{-1 / 6}$ in the case of $D(q)$, we can represent this function as $D=q \cdot\left(f_{1}(q) f_{2}\left(q^{3}\right)\right)^{4}+\left(f_{2}(q) f_{1}\left(q^{3}\right)\right)^{4}$.

Expanding these out, one obtains

$$
C=1+q+2 q^{3}+2 q^{4}+q^{5}+2 q^{6}+2 q^{7}+3 q^{8}+4 q^{9}+O\left(q^{10}\right)
$$

and

$$
D=1+q+2 q^{3}+2 q^{4}+q^{5}+2 q^{6}+2 q^{7}+3 q^{8}+4 q^{9}+O\left(q^{10}\right)
$$

Clearly the identity that we want is $C-D=0$ (in fact $C-D=0+O\left(q^{100}\right.$ ) when expanded out in Pari). This computation establishes the first of the modular equations listed above.

\section{Additional Weber Modular Equations}

We complete this paper by listing some modular equations of the irrational kind that Weber himself considered. These equations are ones which Weber was not able to compute himself and represent gaps in his table, but which we are able to give due to the computing technology now available.

Firstly, let

$$
\begin{gathered}
u=\mathfrak{f}(\tau), \quad v=\mathfrak{f}\left(\frac{a \tau+b}{d}\right), \\
u_{1}=\mathfrak{f}_{1}(\tau), \quad v_{1}=\left(\frac{2}{d}\right) \mathfrak{f}_{1}\left(\frac{a \tau+b}{d}\right), \\
u_{2}=\mathfrak{f}_{2}(\tau), \quad v_{2}=\left(\frac{2}{a}\right) \mathfrak{f}_{2}\left(\frac{a \tau+b}{d}\right),
\end{gathered}
$$

where the definitions of $v_{1}$ and $v_{2}$ involve Kronecker symbols.

Weber's own analysis leads him to define functions $A$ and $B$, depending on the degree $m$ of the modular equation, as follows:

For $m \equiv 7(\bmod 8)$ :

$$
\begin{aligned}
2 A & =u v+(-1)^{\frac{n+1}{8}}\left(u_{1} v_{1}+u_{2} v_{2}\right), \\
B & =\frac{2}{u_{1} v_{1}}+\frac{2}{u_{2} v_{2}}+(-1)^{\frac{n+1}{8}} \frac{2}{u v}
\end{aligned}
$$

For $m \equiv 3(\bmod 8)$ :

$$
\begin{aligned}
& 4 A=u^{2} v^{2}-u_{1}^{2} v_{1}^{2}-u_{2}^{2} v_{2}^{2}, \\
& B=\frac{4}{u_{1}^{2} v_{1}^{2}}+\frac{4}{u_{2}^{2} v_{2}^{2}}-\frac{4}{u^{2} v^{2}} .
\end{aligned}
$$

For $m \equiv 1(\bmod 4)$ :

$$
\begin{aligned}
& 8 A=u^{4} v^{4}-u_{1}^{4} v_{1}^{4}-u_{2}^{4} v_{2}^{4}, \\
& B=\frac{16}{u_{1}^{4} v_{1}^{4}}+\frac{16}{u_{2}^{4} v_{2}^{4}}-\frac{16}{u^{4} v^{4}} .
\end{aligned}
$$

We compute modular equations missing from Weber, up to and including $m=21$, after which, they begin to become unwieldy. Since Weber has already done all the analysis for us, all that remains is to cause the $q$-series to vanish with the computer. We obtain the following modular equations, again after considerable operator time, but little actual computation time.

$m=9$ :

$$
A^{6}-A B-14 A^{3}-3=0
$$


$m=13$ :

$$
A^{7}-B^{2}-22 A^{2} B-105 A^{4}+64 A=0 .
$$

$m=17$ :

$$
A^{3}-B-10 A^{2}+13 A+12=0 .
$$

$m=21$ :

$$
\begin{gathered}
A^{16}-A^{5} B^{4}-38 A^{7} B^{3}-481 A^{9} B^{2}-2222 A^{11} B-2572 A^{13} \\
-10 A^{4} B^{3}+435 A^{6} B^{2}+25106 A^{8} B+251486 A^{10}-35 A^{3} B^{2}+6054 A^{5} B \\
+119716 A^{7}+B^{2}-10 A^{2} B+20809 A^{4}-576 A=0 .
\end{gathered}
$$

\section{REFERENCES}

[1] Bruce Berndt, Ramanujan's Notebooks Part III, Springer-Verlag, 1991.

[2] Bruce Berndt, Heng Huat Chan, Liang-Cheng Zhang Ramanujan's class invariants, Kronecker's limit formula, and modular equations. Trans. Amer. Math. Soc. 349 (1997), no. 6, pp. 2125-2173.

[3] Heng Huat Chan, Wen-Chin Liaw On Russell-type modular equations. Canad. J. Math. 52 (2000), no. 1, pp. 31-46.

[4] Chris Cummins, Sebastian Pauli Congruence subgroups of PSL(2,Z) of genus up to 24 Experimental Mathematics 12, no. 2, (2003), pp. 243-255.

[5] K. G. Ramanathan Ramanujan's modular equations. Acta Arith. 53 (1990), no. 4, pp. 403-420.

[6] B. Schoeneberg Elliptic Modular Functions Die Grund. der Math. Wiss. 203. SpringerVerlag 1974.

[7] William Hart, Class invariants from a new kind of Weber-like modular equation Preprint.

[8] William Hart Explicit evaluation of the Dedekind eta function PhD. Thesis, 2004 , Macquarie University, Sydney.

[9] William Hart Schlaefli modular equations for generalized Weber functions To appear in the Ramanujan Journal.

[10] Heinrich Weber Lehrbuch der Algebra, Dritter Band, Friedrich Vieweg und Sohn, Braunschweig, 1908; reprinted by Chelsea, New York, 1961.

[11] Heinrich Weber Zur theorie der elliptischen funktionen Acta Math. 11 (1888), pp. 333-390.

E-mail address: wbhart@math.uiuc.edu 